\title{
A citar se aprende citando: experiencia didáctica en alfabetización académica
}
(One Learns to Quote by Quoting: Didactic Experience in Academic Literacy)

\section{Elia Saneleuterio Temporal ${ }^{(\mathbb{*}}$, Universitat de V alència, València, España}

\section{Volumen 2, número 1}

Junio 2017

p. 143-159

Este número se publicó el 17 de junio de 2017

Artículo recibido: 16 de febrero 2017

Artículo Aprobado: 18 de mayo de 2017

ISSN: 2448-5942, doi: https://doi.org/10.36799/el.v2i1.44

\section{Cómo citar}

Saneleuterio, E. (2017). A citar se aprende citando: experiencia didáctica en alfabetización académica. Estudios גambda. Teoría y práctica de la Didáctica en Lengua y Literatura. , 2(1), 143-159. https://doi.org/10.36799/el.v2i1.44

Derechos de autor: El autor o autores conservan en todo momento sus derechos morales y patrimoniales sobre la obra; la obra no se puede alterar, transformar o ampliar; siempre debe reconocerse la autoría del documento referido. Ninguna de las modalidades de los documentos publicados en Estudios $\lambda a m b d a$. Teoría y práctica de la didáctica en lengua y literatura tienen fines comerciales de naturaleza alguna.

Los contenidos de este artículo están bajo una licencia de Creative Commons Atribución No Comercial- Sin Derivadas 4.0 Internacional $\oplus \oplus$ 


\title{
A citar se aprende citando: experiencia didáctica en alfabetización académica
}

\author{
To quote is learned by quoting: didactic experience in academic literacy
}

ELIA SANELEUTERIO ${ }^{1}$

\section{RESUMEN}

Ante el problema relativamente extendido del plagio involuntario, nos plantemos la necesidad de fortalecer en el alumnado las competencias implicadas a la hora de insertar párrafos o ideas de otros autores en sus trabajos académicos. La presente experiencia didáctica es el resultado de varias fórmulas previas probadas con los estudiantes.

Metodológicamente, para su análisis, nos valemos de un diseño de investigación acción, de manera que la observación y las respuestas de un cuestionario conducen a unas conclusiones que confirman el éxito de la experiencia.

Esta se llevó a cabo con 212 estudiantes que inician su formación universitaria para ser maestros de Educación Primaria o Infantil. Partió de una fase de concienciación sobre la propiedad intelectual y la honestidad académica, a la que siguió el intercambio de percepciones sobre algunas de las convenciones de este género discursivo.

Se practicaron algunas técnicas de redactado y maquetación de las citas y listados, combinando actividades activas y pasivas relacionadas con sus tipologías. Asimismo, la práctica presencial en el aula se complementó con el trabajo colaborativo virtual mediante los foros de la plataforma institucional de la universidad.

El diseño está enfocado para incidir especialmente sobre los errores habituales entre el alumnado de grado, especialmente acerca del uso de paréntesis para las referencias, así como en la adecuada redacción de las mismas en el apartado final. Asimismo, determinamos que, para optimizar el aprendizaje, el proceso debe comprender la retroalimentación inmediata y preferiblemente abierta, tanto presencial como virtual.

PALABRAS CLAVE: Docencia universitaria, elaboración de trabajos académicos, citación bibliográfica, listado de referencias.

\begin{abstract}
Faced with the relatively widespread problem of involuntary plagiarism, there is the need to strengthen in students some competences involved in inserting paragraphs or ideas of other authors in their academic papers. The present experience is the result of several previous formulas tested with students.

For the analysis of its method, an action research design is used, so that the observation and the answers of a questionnaire lead to conclusions that confirm the success of the experiment.

The present research project was carried out with 212 students who began their university education to be teachers in elementary school.. It started from a phase of awareness about intellectual property and academic honesty, followed by the exchange of perceptions about some of the conventions of this discursive genre.

Some techniques of writing and layout of citations and lists were practiced, combining active and passive activities related to their typologies. Likewise, classroom practices were complemented by virtual collaborative work through the forums of the university institutional platform.

The design is focused to concentrate especially on the common mistakes among pre-graduate students, especially on the parentheses for reference uses, and their proper writing as well in the final section. One of the conclusions, in order to optimize learning, is that the process must incorporate immediate and preferably open feedback, both face-to-face and virtual.
\end{abstract}

KEYWORDS: University teaching, academic papers composition, bibliographic citation, list of references.

\section{INTRODUCCIÓN}

\footnotetext{
${ }^{1}$ Elia Saneleuterio es doctora en Literatura Española por la Universitat de València, licenciada en Filología Hispánica, maestra en Educación Infantil y máster en Investigación en Didácticas Específicas. Es profesora ayudante doctora en el Departament de Didàctica de la Llengua i la Literatura de la Universitat de València (España). elia.saneleuterio@uv.es

Artículo recibido: 16 de febrero 2017

Artículo enviado a corrección: 17 de abril de 2017

Aprobado: 18 de mayo de 2017

Saneleuterio Temporal doi: https://doi.org/10.36799/el.v2i1.44 Volumen 2, número 1, Año 2017, ISSN: 2448-5942
} 
Dentro del marco del Espacio Europeo de Educación Superior, la propuesta que a continuación se desarrolla se alinea con las directrices que tienen que ver con la superación de la idea del docente como actor principal y único en la transmisión y evaluación del aprendizaje. Además seguimos la estela de las universidades de América del Norte y sus programas de “compañeros de escritura en las materias" (Carlino, 2005), así como la creciente preocupación sobre alfabetización académica que, desde centros de escritura y universidades de todo el mundo, han dado lugar a propuestas, experiencias y metodologías que son guía y precedente de esta (Carlino, 2005; Heredia y Quirós, 2015; Muse y Delicia, 2015; Núñez Cortés, 2014; GómezDevís y Saneleuterio, 2017), sobre todo desde la constatación de que la calidad de los trabajos académicos que entrega el alumnado universitario no siempre es la requerida (Lea y Street, 1998; Zerdán, 2003; Álvarez, Villardón y Yáñiz, 2013). En concreto, este artículo aborda un aspecto fundamental del dominio de la escritura en la mayoría de géneros académicos: el tratamiento de la bibliografía.

El objetivo que se persigue con el estudio del diseño que se propone es demostrar que la participación activa en tareas que requieren citar fuentes bibliográficas está relacionada con dos tendencias: el aumento de la calidad de los trabajos que se realizan, al incorporar base bibliográfica explícita, y la disminución de los casos de plagio, especialmente el que se produce por desconocimiento o falta de competencia. Respecto al deliberado, se entiende que también se reducirá al poner en valor un trabajo que reconoce las aportaciones ajenas frente a un ensayo que no incluye ninguna cita. De todas formas, quienes pretenden un plagio deshonesto, cuya osadía este diseño también pretende reducir de soslayo, quedan disuadidos ante la certeza del suspenso, dada la existencia de escáneres o programas que lo detectan automáticamente al ser depositado el texto en la plataforma virtual de las instituciones que cuentan con este servicio académico.

La propuesta se concreta en el diseño de un seminario que incluyó varias prácticas de citación y que se ha llevado a cabo durante varios cursos académicos con diversos grupos de primero de grado.

Estas prácticas, precedidas de explicaciones de la profesora y de ejemplificaciones animadas disponibles en línea, se centraron en cuestiones técnicas de redactado y maquetación 
de las citas y listados, con especial atención a las normas APA. Se combinaron actividades activas y pasivas relacionadas con sus tipologías.

\section{JUSTIFICACIÓN Y OBJETIVOS}

Ante los problemas de plagio que presentan de manera recurrente los trabajos que parte del estudiantado universitario realiza en las diversas materias, encontramos el factor involuntario, del que se deriva que muchos estudiantes consideran válidos procedimientos y resultados que no lo son en absoluto. En la raíz de estas prácticas hay una falta de competencia para reconocer e insertar ideas ajenas: la experiencia didáctica que analizamos parte de la presentación a los estudiantes universitarios, ya en el primer curso, las normas básicas de respeto y reconocimiento de las ideas o palabras de otras personas, basándonos en sus dos puntos fuertes: estas dan valor a nuestros trabajos académicos y nos ayudan a evitar el plagio. El enfoque práctico de la experiencia, además, aporta a los estudiantes herramientas para adquirir competencia a la hora de citar fuentes y elaborar el listado bibliográfico.

El diseño requiere un mínimo de dos sesiones en un aula de informática, o en el aula habitual del grupo siempre que se garantice que los estudiantes traen al menos un portátil por pareja y tienen acceso a la red wifi de la universidad.

Algunos objetivos que justifican y orientan nuestro enfoque son:

- La necesidad de concienciación acerca de las causas y consecuencias del plagio en la universidad (Marsh, 2007; Gilmore, 2008; Sutherland-Smith, 2008; Saneleuterio, 2012).

- El dominio de las técnicas para insertar citas en el texto y componer un buen listado bibliográfico.

- La identificación de tipologías y la competencia en composición de citaciones.

- La dotación de herramientas y la iniciación en su aprovechamiento para el desarrollo de competencias en escritura académica (Heredia y Quirós, 2015; Gómez-Devís y Saneleuterio, 2015).

Saneleuterio Temporal doi: https://doi.org/10.36799/el.v2i1.44 Volumen 2, número 1, Año 2017, ISSN: 2448-5942 
- La asimilación, en última instancia, de las convenciones del género discursivo académico que tienen que ver con el tratamiento de la bibliografía (Muse y Delicia, 2015).

\section{CONTEXTUALIZACIÓN}

El diseño se puso en práctica en la Universitat de València (España), concretamente en los grados de Maestro o Maestra a en Educación Primaria y de Maestro o Maestra a en Educación Infantil. Los participantes fueron los 212 alumnos matriculados en varios grupos de la asignatura de primer curso Lengua Española para Maestros, durante los cursos 2014-2015 y 2015-2016.

En esta asignatura se trabajó la tipología textual expositiva, y la propuesta lo centró en el género académico del trabajo de investigación, desarrollando un seminario de elaboración de trabajos académicos. Dentro de este, la experiencia descrita se refiere a una de sus partes axiales: la redacción y composición de citas y referencias bibliográficas

\section{DESCRIPCIÓN Y METODOLOGÍA}

\subsection{Fase de concienciación}

En primer lugar partimos de una fase de concienciación sobre dos aspectos fundamentales: el plagio y la importancia de consultar y citar fuentes. Se realiza en el aula de informática con un par de animaciones en línea:

Cómo citar y elaborar referencias bibliográficas: Tutorial de Competencias informáticas e informacionales http://www.ci2.es/objetos-de-aprendizaje/elaborando-referencias-bibliograficas

Estilos de cita: Servicio de Biblioteca Universidad de la Laguna http:/ /www.ull.es/view/ institucional/bbtk/Estilos de cita/es

Estas animaciones fueron complementadas con lluvias de ideas y puesta en común de argumentos que construyan la idea de que no podemos apropiarnos de ideas ni palabras que no sean nuestras, además de que una buena cita aporta calidad a nuestro trabajo.

Saneleuterio Temporal doi: https://doi.org/10.36799/el.v2i1.44 Volumen 2, número 1, Año 2017, ISSN: 2448-5942 


\subsection{Fase teórica}

Así pues, iniciamos una segunda fase, teórica, sobre diferentes aspectos relacionados con la citación. Uno de los puntos teóricos claves fue la distinción de los tipos de fuentes y el modo en que ello se refleja en el listado bibliográfico: vimos principalmente cómo redactar la referencia de un libro, de un capítulo de libro, de un artículo y de un recurso en línea. El contenido sería el que sigue:

\subsubsection{Referencias / Bibliografía citada}

El trabajo debe contener un listado bibliográfico correctamente transcrito, en correspondencia con el estilo de cita seleccionado (en caso de referencias a pie de página, el apartado de "Bibliografía citada" es opcional): orden alfabético por apellido del autor, inclusión de datos requeridos y con

formato común en todas las entradas. Por ejemplo, este sería el estilo APA: Apellidos, Inicial del nombre. (Año). Título del libro. Lugar: Editorial. Apellidos, Inicial del nombre. (Año). Título del artículo. Revista, volumen (número), págs. Apellidos, Inicial del nombre. (Año). Título del capítulo. En Autor, Título del libro (pp. x-y). Lugar: Editorial.

Si están disponibles online se añade el URL (la fecha de consulta ya no es obligatoria). Ejemplo: Recuperado de <htpp://se-copia-y-pega-la dirección*>.

Si se trata de otro tipo de recursos en Internet (la información desconocida se omite):

Apellidos, Inicial del nombre. (Año). Título del documento [en línea]. Nombre de la web. $<$ htpp://se-copia-y-pega-la dirección*>.*Aunque se recomienda usar acorta-enlaces.

Se puede variar la puntuación y tipografía, pero siempre manteniendo la homogeneidad del listado. Asimismo, sobre todo cuando el sistema no es de paréntesis, el año puede ir al final. Ejemplo (menos recomendable que el anterior):

ApELLIDOS, Nombre: Titulo del libro, Lugar, Editorial, año.

APELlidos, Nombre: «Título del artículo», Revista, número, año, páginas.

APELLIDOS, Nombre: «Título del capítulo», en AUTOR, Título del libro, Lugar, Editorial, año, pp.

4.2.2. Sistema coherente de citación. ¿Cómo dar la referencia de cada fragmento citado?

Existen varios sistemas o estilos, que pueden englobarse en dos grandes grupos:

Sistema de paréntesis: junto a cada cita va entre paréntesis el apellido del autor, año y página; la referencia completa va en la bibliografía. Ej.: (Autor, año, p. x) / Autor (año, pp. x-y)

Sistema de referencia a pie de página: en el texto, cada vez que citemos: llamada a nota; en nota: referencia completa (la 1. ${ }^{a}$ vez; luego, ref. abreviada).

Dentro de cada grupo podemos definir nuestro modo de puntuar o seguir el estilo preestablecido de alguna asociación o publicación (hay muchos, con grandes o ligeras variantes entre ellos, como APA, MLA, etc.; véanse los enlaces), pero lo importante es que el trabajo presente homogeneidad en el tratamiento y citación de la bibliografía, es decir, no se puede variar el sistema o estilo de cita dentro en un mismo trabajo. (En Magisterio se usa el sistema de paréntesis, especialmente APA).

Saneleuterio Temporal doi: https://doi.org/10.36799/el.v2i1.44 Volumen 2, número 1, Año 2017, ISSN: 2448-5942 
4.2.3. Tipos de citas. Los estudiantes deben tomar conciencia de que cuando quieran hacer mención de una idea ajena o copiar literalmente unas líneas de otro autor o autora necesitan contextualizarlas y elegir cómo quedarán mejor en su texto. Así pues, el tipo de cita depende de cómo introducimos en cada momento la información que citamos, siendo recomendable que los variemos para evitar la monotonía. En este punto se les advirtió de no confundir "tipos de citas" con "sistema o estilo de citación”, que sí ha de ser el mismo en todo el trabajo.

Las citas indirectas o parafraseadas captan la idea del autor, pero cambiamos palabras, modificamos el orden, resumimos, etc. Por ello, no necesitan comillas ni ninguna otra marca, pero sí la referencia (entre paréntesis o a pie de página, según el sistema elegido). En caso contrario, se considera plagio.

Las citas directas o textuales (copiadas literalmente del autor) presentan dos formas de insertarse en el texto, dependiendo de su extensión.

Si es breve, va entrecomillada dentro del texto (como en los ejemplos de arriba).

Si es larga (3 líns. o más), se recomienda sacarla del cuerpo del texto, es decir, transcribirla en párrafo aparte. Para marcar tipográficamente que se trata de palabras ajenas se sangra el párrafo y se reduce la letra (de esta forma, no hacen falta las comillas). 


\subsubsection{Ejemplos}

\begin{tabular}{|c|c|c|}
\hline & Sistema paréntesis (ejemplo APA) & Sistema de referencia a pie de página \\
\hline Indirecta o & \multirow{4}{*}{$\begin{array}{l}\text { Los poetas, cuando escriben sobre otros poetas, } \\
\text { suelen hacerlo - consciente o inconscientemente- } \\
\text { para justificarse a sí mismos (Rosell, 2009, p. 92). } \\
\text { Miquel Oltra (2013) analiza las características } \\
\text { esenciales del teatro de marionetas y plantea } \\
\text { algunas propuestas didácticas interesantes. }\end{array}$} & \multirow{4}{*}{$\begin{array}{l}\text { Los poetas, cuando escriben sobre otros poetas, } \\
\text { suelen hacerlo - consciente o } \\
\text { inconscientemente- para justificarse a sí } \\
\text { mismos }^{1} \text {. } \\
\text { Miquel Oltra }{ }^{2} \text { analiza las características } \\
\text { esenciales del teatro de marionetas y plantea } \\
\text { algunas propuestas didácticas interesantes. }\end{array}$} \\
\hline parafra- & & \\
\hline$\underline{\text { seada }}$ & & \\
\hline $\begin{array}{l}\text { La expresamos } \\
\text { con nuestras } \\
\text { palabras. }\end{array}$ & & \\
\hline textual & $\begin{array}{l}\text { Según María Rosell, la crítica ejercida por poetas } \\
\text { acaba siendo una "vía propicia para la justificación } \\
\text { personal" (2009, p. 92). }\end{array}$ & $\begin{array}{l}\text { Según María Rosell, la crítica ejercida por poetas } \\
\text { acaba siendo una "vía propicia para la } \\
\text { justificación personal"3. }\end{array}$ \\
\hline $\begin{array}{l}\text { La transcribi- } \\
\text { mos literal y } \\
\text { entrecomillada, } \\
\text { si es breve. }\end{array}$ & $\begin{array}{l}\text { Podemos estar de acuerdo en que un rasgo esencial } \\
\text { del teatro de marionetas es "el mestissatge de formes } \\
\text { i de tècniques de manipulació" (Oltra, 2013, p. 203). }\end{array}$ & $\begin{array}{l}\text { Podemos estar de acuerdo en que, en el teatro } \\
\text { de marionetas, un rasgo esencial es "el mestis- } \\
\text { satge de formes i de tècniques de manipulació" }\end{array}$ \\
\hline \multirow[t]{2}{*}{$\begin{array}{l}\text { Referencias } \\
\text { completas: }\end{array}$} & \multirow[t]{2}{*}{$\begin{array}{l}\text { En el listado bibliográfico aparecerá: } \\
\text { OLTRA ALLACH, M. À. (2013). Els titelles, eing. d'educació } \\
\text { literaria i intercultural. València: Perifèric. } \\
\text { ROSELL, M. (2009). La historia es de los poetas: tradición y } \\
\quad \text { crítica en algunas voces del } 27 \text {. Olivar, 13,83-95. }\end{array}$} & 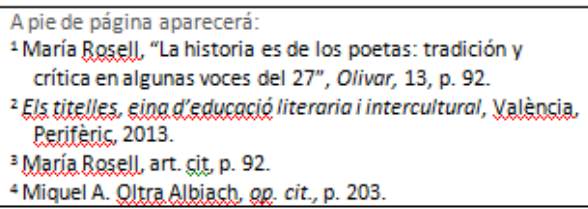 \\
\hline & & $\begin{array}{l}\text { [Listado bibliográfico opcional, porque las referencias } \\
\text { completas ya se han dado a pie de página] }\end{array}$ \\
\hline
\end{tabular}

Fig. 1. Ejemplos de citas pafraseadas y directas breves 


\begin{tabular}{|c|c|}
\hline \multicolumn{2}{|l|}{$\begin{array}{r}\text { Citas sangradas } \\
\text { Son citas directas o textuales transcritas en un párraf }\end{array}$} \\
\hline $\begin{array}{l}\text { Son citas directas o textuales transcritas en un } \\
\text { Mediante el sistema de paréntesis (ejemplo APA) }\end{array}$ & $\begin{array}{l}\text { aparte debido a su extensión. } \\
\text { Mediante el sist. de pie de página }\end{array}$ \\
\hline $\begin{array}{l}\text { El maestro proporcionará material y los alumnos } \\
\text { evaluarán la adecuación del uso del masculino para } \\
\text { designar a seres de ambos géneros. Sonia Bailini (2012, p. } \\
\text { 80) deja bastante claro cómo debe actuar todo profesor } \\
\text { en actividades de estetipo: } \\
\text { El primero tiene que estar dispuesto a ceder su rol de prota- } \\
\text { gonista para convertir la tarea docente en una actividad } \\
\text { coral en la que él desempeña el papel de director de escena } \\
\text { [...]. } \\
\text { Para analizar el uso de expresiones como "tus hijos e } \\
\text { hijas", "los alumnos y las alumnas", etc., habremos de } \\
\text { tener en cuenta que la duplicación solo se admite en } \\
\text { casos de posible confusión o en ámbitos muy concretos, } \\
\text { como el político o el escolar. } \\
\text { Exceptuados estos usos, el circunloquio es innecesario } \\
\text { cuando el empleo del género no marcado es } \\
\text { suficientemente explícito para abarcar a los individuos de } \\
\text { unoy otro sexo. (RAEy AALE, 2009,p. } 87 \text { ) }\end{array}$ & $\begin{array}{l}\text { El maestro proporcionará material y los } \\
\text { alumnos evaluarán la adecuación del uso } \\
\text { del masculino para designar a seres de } \\
\text { ambos géneros. Sonia Bailini deja bastan- } \\
\text { te claro cómo debe actuar todo profesor } \\
\text { en actividades de este tipo: } \\
\text { El primero tiene que estar dispuesto a } \\
\text { ceder su rol de protagonista para con- } \\
\text { vertir la tarea docente en una actividad } \\
\text { coral en la que él desempeña el papel de } \\
\text { director de escena [...]. }{ }^{1} \\
\text { Como punto de partida cabría considerar } \\
\text { que la Nueva gramática de la lengua } \\
\text { española de la Real Academia Española }{ }^{2} \\
\text { recomienda reservar duplicaciones inne- } \\
\text { cesarias a ámbitos muy concretos, como } \\
\text { el político o el escolar: } \\
\text { el circunloquio es innecesario cuando el } \\
\text { empleo del género no marcado es sufi- } \\
\text { cientemente explícito para abarcar a los } \\
\text { individuos de uno y otro sexo. } \\
{ }^{1} \text { Sonia Bailini, "Hacia un tratamiento profesional del } \\
\text { texto: prácticas para el desarrollo de la } \\
\text { competencia discursiva", Lenguaje y textos, } 36 \text {, } \\
\text { noviembre, 2012, pp. } 73-81 \text {. } \\
{ }^{2} \text { Vol. 1, Madrid, Espasa, 2009, p. } 87 \text {. }\end{array}$ \\
\hline & bibliográfico on \\
\hline
\end{tabular}

Fig. 2. Ejemplos de citas directas largas o sangradas

Los ejemplos mostrados en las figuras 1 y 2 se completaron con los aportados en las animaciones citadas arriba:

- Cómo citar y elaborar referencias bibliográficas: Tutorial de Competencias informáticas e informacionales http://www.ci2.es/objetos-de-aprendizaje/elaborandoreferencias-bibliograficas.

- Estilos de cita: Servicio de Biblioteca Universidad de la Laguna http://www.ull.es/ view/institucional/bbtk/Estilos de cita/es

\subsection{Fase práctica}

Saneleuterio Temporal doi: https://doi.org/10.36799/el.v2i1.44 Volumen 2, número 1, Año 2017, ISSN: 2448-5942 
Para la fase práctica, se propusieron diferentes actividades, combinando actividades virtuales de identificación y análisis con otras de síntesis y creación, para lo que se trabajó tanto con publicaciones en papel como en línea. Las prácticas se facilitaron en formato $W$ ord, para que pudieran colorear o realizar las propuestas sobre el mismo documento de texto. Como veremos, con ellas los estudiantes se entrenaron en discriminación de tipologías bibliográficas, en varias formas de citación textual y paratextual, mediante sistemas de paréntesis, así como en la adecuada redacción de las referencias finales, llamando la atención en los errores habituales entre el alumnado de grado.

4.3.1. Actividad de creación y detección de dificultades. Mediante metodología cooperativa, los estudiantes se repartieron las tipologías de citas y, centrándose en una sola, la explicaron por parejas rellenando una ficha virtual que incluía un ejemplo creado por ellos. Cada pareja compartió su aportación en el foro de la asignatura, de manera que los compañeros pudieron comentar errores o dudas y la retroalimentación resultó accesible para todos. Veamos una de las conversaciones:

2: Re: Res: Práctica de citas (respuesta a 1 )

Enviado por Elia Saneleuterio Temporal on 04/11/14 07:51

Hay algunos problemas... ¿Quién los identifica?

3: Re: Res: Práctica de citas (respuesta a 1)

Enviado por Andrea *** on 07/11/14 12:13

Creo que uno de los errores es al mencionar el autor, el año y la página de la obra, lá página debería ir dentro del paréntesis al igual que el año después de dos puntos. Sería así: "Como dice Torvarelli (2013:38)..."

4: Re: Res: Re: Res: Práctica de citas (respuesta a $\underline{3}$ )

Enviado por Andrea *** on 07/11/14 12:14

$*$ la, sin acento(corrección)

5: Re: Res: Re: Res: Práctica de citas (respuesta a $\underline{3}$ )

Enviado por Elia Saneleuterio Temporal on 10/11/14 10:26

Bien. Falta otra cosa (además de los espacios entre algunas palabras): fijaos en el título de la revista...

6: Re: Res: Re: Res: Re: Res: Práctica de citas (respuesta a $\underline{5}$ )

Enviado por Antonio *** on 27/11/14 13:12

El título debería estar en letra cursiva.

Saneleuterio Temporal doi: https://doi.org/10.36799/el.v2i1.44 Volumen 2, número 1, Año 2017, ISSN: 2448-5942 
4.3.2. Actividad de reconocimiento. A continuación, se planteó una práctica de reconocimiento, que también debían compartir en el foro una vez resuelta, aunque la corrección se hizo en clase, proyectando una de las aportaciones (figura 3), discutiendo las opciones y comentándolas.

II. Identifique en el ejemplo citas textuales sangradas, entrecomilladas y citas indirectas o parafraseadas, notas al pie para comentarios o aclaraciones, notas al pie para referencias, realzado de términos, plurales de modestia, incisos entre rayas o paréntesis, incisos entre comas. Márquelo con distintos colores.

La imagen, como sabemos, está intrínsecamente relacionada a la expresión poética, hasta el punto de considerarse un elemento poético imprescindible, pues en ella reside la magia del poema: nada puede explicarla o parafrasearla de manera fiel, su sentido es ella misma. «La imagen se explica a sí misma. Nada, excepto ella, puede decir lo que quiere decir» (Paz 1967: 109-110). La particularidad de la imagen va incluso más allá: su función primordial es dotar de intensidad al poema, es decir, transformarlo en palabra poética. Pero esta capacidad no le viene de su imposibilidad de ser parafraseada, sino, como bien defiende y explica Jean Cohen, de su imposibilidad de ser negada.

Privada de oposición, la palabra se afirma como TOTALIDAD semántica y se otorga un sentido absoluto. Este es el «sentido más puro» que la poesía da a las palabras de la lengua. Más puro por estar depurado de lo que niega en el uso que de ella hace la tribu. (1982: 103)

Pero Cohen no se detiene ahí: esta imposibilidad de encontrar opuesto es lo que hace del lenguaje poético un lenguaje estructuralmente totalizador y funcionalmente intensificador. La figura poética es la que, según el teórico, transforma el concepto en imagen mediante una operación de cambio no en el contenido, sino en la forma del sentido: por eso la imagen poética se expresa siempre en un lenguaje afectivo ${ }^{1}$. Así, partiendo del papel de la imagen, desentraña este estudioso el lenguaje de la poesía

1 Vid. Jean Cohen, El tenguaje de la poesía, Teoría de la poeticidad, Madrid, Gredos. 1982, págs.126-132.

${ }^{2}$ La teoría de la poeticidad de Cohen sostiene, en resumen, que el lenguaje de la poesía es intenso porque es totalizador, cosa que se deriva del hecho de que no tiene opuesto — por eso precisamente es un lenguaje desviado—. Y es así que la poesía intenta expresar una experiencia también intensa y totalizadora.

Fig. 3. Práctica de reconocimiento

\subsubsection{Actividad de reflexión. El párrafo propuesto presentaba algunos problemas} intencionados, como ítems sin ejemplo o falta de homogeneidad. Sobre ello, así como sobre qué referencias podríamos esperar, versaban las siguientes preguntas de la práctica (véase, por ejemplo, la IV en la figura 4), que se contestaron por parejas y se debatieron o clarificaron en clase, dado que era esperable que no todas las parejas consiguieran resolverlo adecuadamente de manera autónoma.

IV. Si elegimos el sistema de paréntesis, debe ir al final del documento un listado de bibliografía citada. Para la referencia primera cita del fragmento anterior, ¿cuáles serían la opciones válidas? ¿Por qué?

a) Paz, Octavio, El arco y la lira, México, Fondo de Cultura Económica, 1967.

b) Paz, Octavio (1967): El arco y la lira, México, Fondo de Cultura Económica [1. a ed.: 1956].

c) Paz, Octavio (1975): Los hijos del limo, Barcelona Seix Barral.

d) Paz Gómez, José C. (1967): “En torno a la problemática foral”, Revista de economía de Galicia, 57-60, pp. 20-24.

e) Paz Gómez (1967): La imagen, Barcelona, Anthropos.

f) Martínez, Paz (1967): “La imagen poética”, Cuadernos de poesía, III, pp. 109-112.

Fig. 4. Actividad de reflexión

Saneleuterio Temporal doi: https://doi.org/10.36799/el.v2i1.44 Volumen 2, número 1, Año 2017, ISSN: 2448-5942 
4.3.4. Actividad de creación de contextos. Finalmente, se planteó una actividad abierta en la que se les facilitó información diversa. Ellos debían redactar contextos que les permitieran citarla de varias maneras (cita indirecta, directa breve o sangrada), así como componer el listado bibliográfico correspondiente. Tras experiencias previas, considero importante, para las áreas de Educación, al menos en las universidades españolas, centrarse en estilo APA en las prácticas, así como afinar en el rigor de las fuentes seleccionadas. Para universidades de otros países, habría que sustituir los ejemplos y prácticas de estilo paréntesis por las normas más habituales en su tradición, por ejemplo MLA.

\section{RESULTADOS: VALORACIÓN Y PERCEPCIÓN DE UTILIDAD}

Tras la experiencia, la fase de investigación se completa con el análisis de los datos recogidos, cualitativamente mediante los trabajos, comentarios en el foro, mensajes de correo electrónico... Y cuantitativamente mediante las respuestas a un cuestionario online (figura 5).

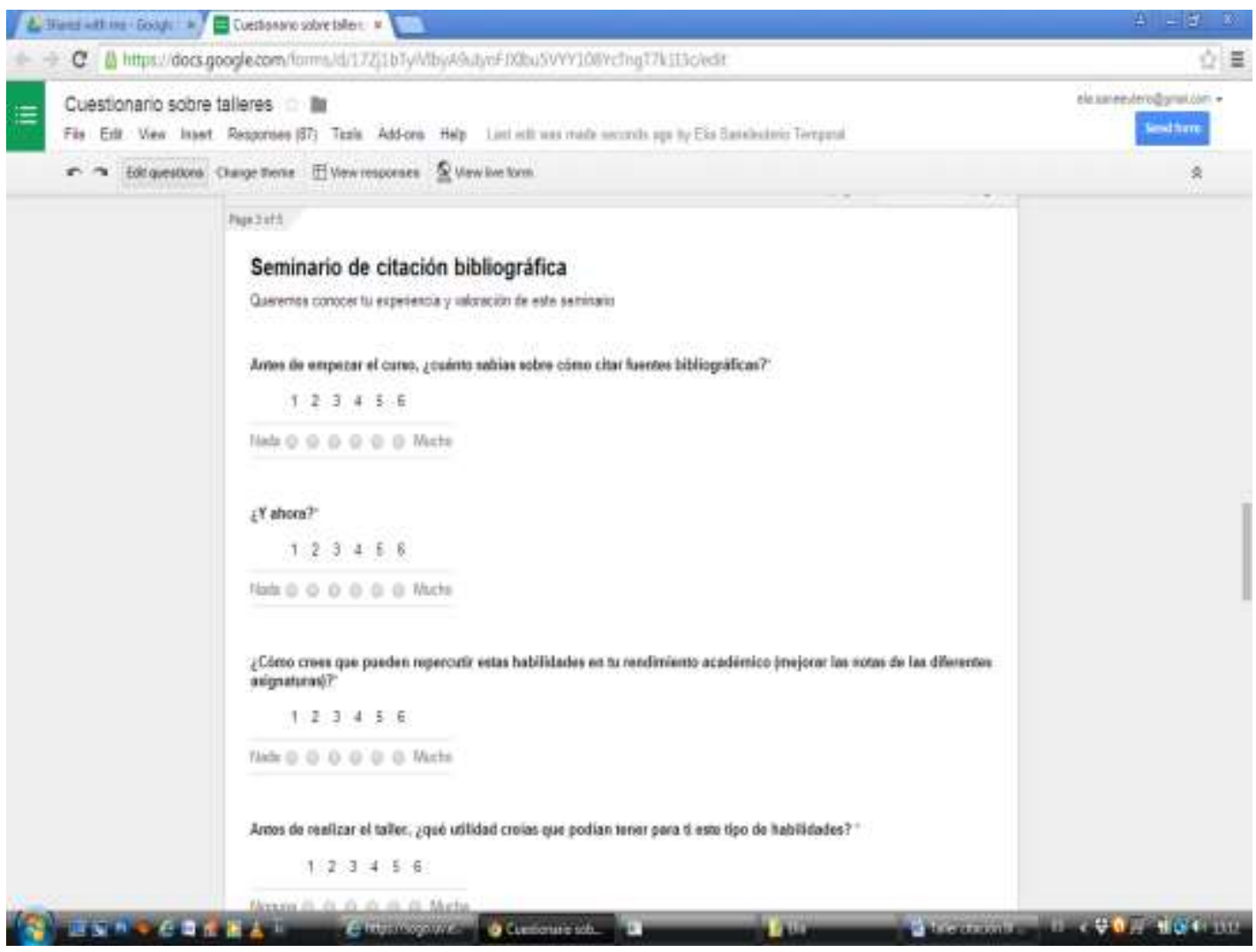

Fig. 5. Cuestionario online de valoración de la experiencia.

Saneleuterio Temporal doi: https://doi.org/10.36799/el.v2i1.44 Volumen 2, número 1, Año 2017, ISSN: 2448-5942 
Las gráficas que siguen (figuras 6 y 7) muestran los resultados correspondientes a las respuestas de dos grupos (84 respuestas en total). Hablaremos de algunos ítems; otros se analizan más detalladamente en Saneleuterio (2015).

Los primeros resultados tienen que ver con la autoconciencia de conocimiento o competencia en citación bibliográfica. Son los correspondientes a las preguntas sobre cuánto sabían sobre cómo citar fuentes bibliográficas antes y después del taller, y que debían ser valoradas en una escala de 1 a 6 . Como se aprecia en la figura 6 , la percepción de la cantidad de conocimientos aprendidos es satisfactoria si comparamos en antes (serie 1) con el después (serie 2).

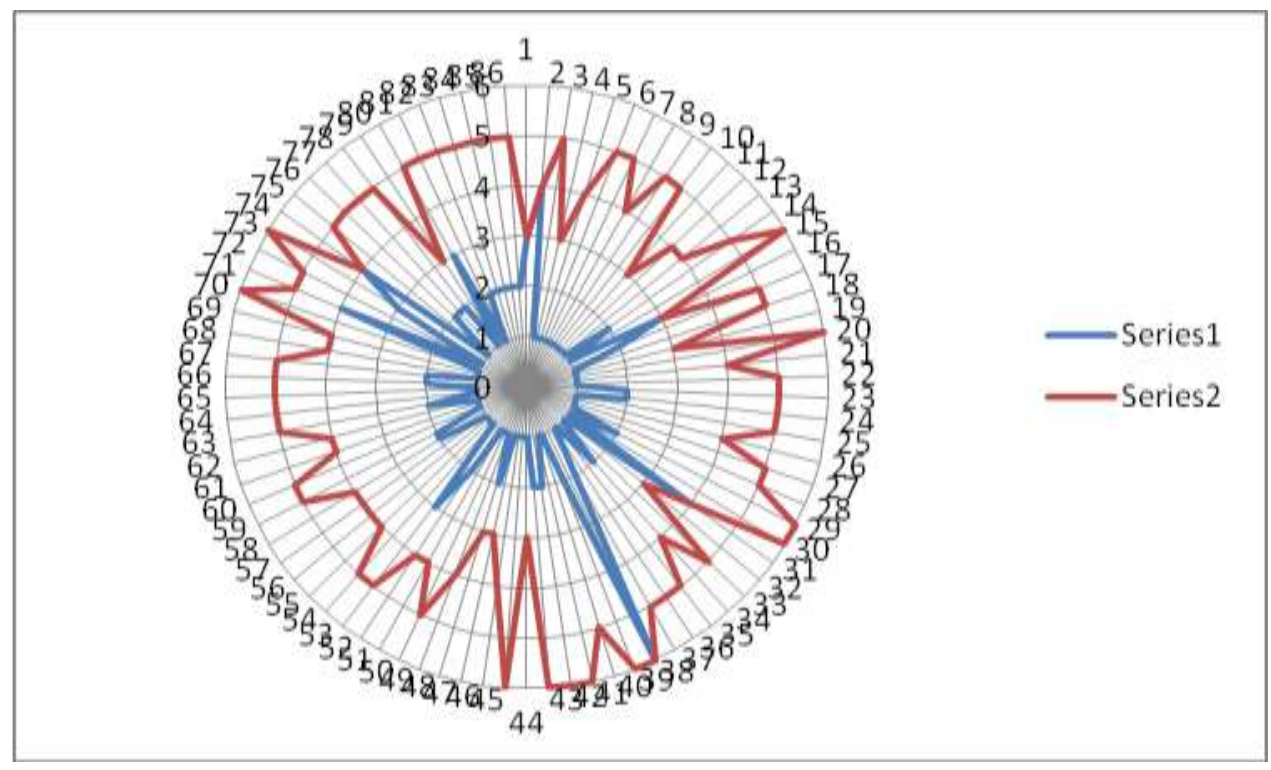

Fig. 6. Autoconciencia de conocimiento o competencia en citación bibliográfica antes/ahora.

Respecto de la utilidad que le atribuyeron a lo aprendido en el taller, se cuantificó en una escala de 1 a 6 a partir de las siguientes preguntas:

- ¿Cómo crees que pueden repercutir estas habilidades en tu rendimiento académico (mejorar las notas de las diferentes asignaturas)?

- Antes de realizar el taller, ¿qué utilidad creías que podían tener para ti este tipo de habilidades? 
Si lo medimos en porcentaje, destaca que frente al 63,1\% de alumnos que reconocen atribuirle poca o ninguna utilidad a la habilidad de hacer un uso adecuado de la bibliografía antes del taller, pasamos al 91,7\% de alumnos que, tras el taller, lo consideran útil o muy útil: en concreto, el 76,2\% se situarían en los puntos cinco y seis de valoración (figura 7). El contraste entre las líneas es representativo del alcance de la sensibilización lograda mediante el taller de citación bibliográfica.

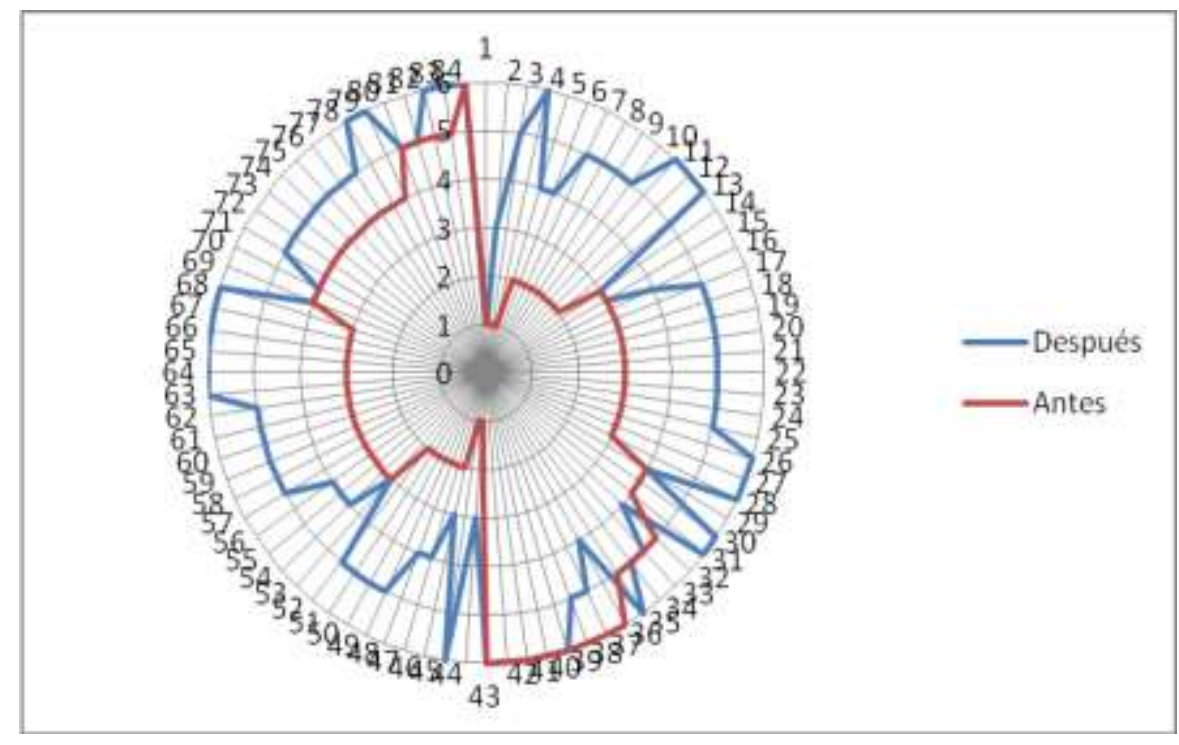

Fig. 7. Utilidad de la competencia en citación bibliográfica antes/ahora.

\section{CONCLUSIONES}

El estudio basado en una estadística valorativa y en la observación cualitativa del desempeño nos lleva a concluir la eficacia del planteamiento. Queda cumplido el objetivo de aumentar la autoconciencia sobre la propia competencia, todavía en desarrollo, pero avanzada con respecto al inicio del curso. Este resultado se mide como percepción y se confirma con el número medio de referencias en los trabajos, así como su más que aceptable calidad y adecuación a las normas APA, estilo predominante en la titulación y en la zona geográfica donde tuvo lugar la experiencia. Podemos sintetizar los hallazgos más relevantes en seis puntos:

- Resulta necesario, para concienciar a los estudiantes acerca de la importancia de la fundamentación bibliográfica de un trabajo académico y su formato, prever en una asignatura de primer curso de grado una acción pedagógica específica.

Saneleuterio Temporal doi: https://doi.org/10.36799/el.v2i1.44 Volumen 2, número 1, Año 2017, ISSN: 2448-5942 
- La propuesta analizada es viable en cuanto al tiempo, y suficientemente abarcadora o profunda para producir aprendizaje: al incorporar ejemplos dinámicos y práctica supone más que una mera introducción.

- Las carencias en cuestiones de tratamiento de la bibliografía son reconocidas por el alumnado, y tras el contacto perciben un aumento de conocimiento al respecto.

- El diseño y la metodología empleada aumentan la conciencia de qué es plagio y la motivación por aprender a citar y referenciar correctamente la bibliografía.

- Asimismo, también aumenta el conocimiento de su trascendencia y utilidad para sus estudios en la universidad, de lo que se muestran muy poco conscientes antes de la experiencia.

- En definitiva, al resultar una experiencia centrada en el conocimiento de qué, cómo y por qué, podemos concluir que el diseño facilita la percepción de utilidad por parte del alumnado.

Cabe señalar, para terminar, que diseños como el expuesto y analizado en este artículo deben considerarse como un paso de un proceso mucho más complejo, la alfabetización académica, que para la selección o consideración de las fuentes y referencias debería complementarse con talleres de búsquedas bibliográficas (Saneleuterio, 2014) que incidan en el rigor de la información citable.

\section{REFERENCIAS BIBLIOGRÁFICAS}

Álvarez, M., Villardón, M. L. y, Yániz, C. Influencia de factores sociocognitivos en la calidad de la escritura en los estudiantes universitarios. Educatio Siglo XXI, 28.2 (2010): 181-203.

Carlino, Paula. Alfabetización académica diez años después. Revista mexicana de investigación educativa, 18.57 (2013): 355-381. Recuperado de: http://www.scielo.org.mx/pdf/rmie/ v18n57/v18n57a3.pdf.

Carlino, Paula. Representaciones sobre la escritura y formas de enseñarla en universidades de América del Norte. Revista de Educación, 336 (2005): 143-168.

Saneleuterio Temporal doi: https://doi.org/10.36799/el.v2i1.44 Volumen 2, número 1, Año 2017, ISSN: 2448-5942 
Competencias informáticas e informacionales. Cómo citary elaborar referencias bibliográficas. Tutorial (s/d). Recuperado de http://www.ci2.es/objetos-de-aprendizaje/elaborandoreferencias- bibliograficas

Gilmore, B. Plagiarism: Why it Happens, How to Prevent it. Heinemann, 2008.

Gómez-Devís, M. ${ }^{a}$ Begoña., y Saneleuterio, Elia. Con la evaluación también se aprende. Percepciones y grado de satisfacción del alumnado en formación inicial en magisterio. En Aguilar López, Ana María y Peña Acuña, Beatriz (coords.), Didáctica de la lengua y la literatura: Buenas Prácticas docentes, vol. II. Madrid: ACCI, 2017: 167-192.

Gómez-Devís, M. ${ }^{a}$ Begoña., y Saneleuterio, Elia. E-evaluación orientada al aprendizaje: diseño para su implementación en la materia Lengua para Maestros. En R. Pérez Pérez, A. Rodríguez-Martín y E. Álvarez-Arregui (eds.), Innovación en la Educación Superior: desafíos y propuestas. Oviedo: Ediciones de la Universidad de Oviedo, 2015: 319-326.

Heredia Ponce, Hugo, y Fátima Quirós Rivero. El taller de escritura académica como estrategia de alfabetización para la creación de textos específicos: memorias de prácticas y trabajos fin de grados. En Xaquín Núñez, Aránzazu González, Carlos Pazos y Pedro Dono (eds.), Horizontes científicos y planificación académica en la Didáctica de Lenguas y Literaturas. Ribeirão: Húmus, 2015: 443-458.

Lea, M. R. y Street, B. V. Student writing in higher education: an academic literacies approach. Studies in Higher Education 23(2) (1998): 157-172.

Marsh, B. Plagiarism: Alchemy and Remedy in Higher Education. Nueva York: SUNY Press, 2007.

Muse, Cecilia E., y Delicia Martínez, Darío. Los géneros discursivos académicos. En Núñez Cortés, J. A. (coord.). Escritura académica: de la teoría a la práctica. Madrid: Pirámide, 2015: 85-101.

Núñez Cortés, Juan Antonio (2014). Alfabetización académica y competencia comunicativa en educación superior. UNAH Innova, 3 (2014): 16-20.

Núñez Cortés, Juan Antonio. Escribir en la universidad. En J. A. Núñez Cortés (coord.), Escritura académica. De la teoría a la práctica. Madrid: Pirámide, 2014: 17-34.

Saneleuterio Temporal doi: https://doi.org/10.36799/el.v2i1.44 Volumen 2, número 1, Año 2017, ISSN: 2448-5942 
Núñez Cortés, Juan Antonio. Las dimensiones de la alfabetización en la educación superior:

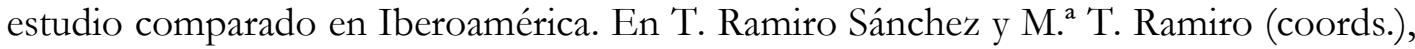
Avances en Ciencias de la Educación y del Desarrollo 2016. Granada: AEPC, 2016: 698-703

Saneleuterio, Elia. Aprendiendo la escritura académica: las referencias. En R. Pérez Pérez, A. Rodríguez-Martín y E. Álvarez-Arregui (eds.), Innovación en la Educación Superior: desafíos y propuestas. Oviedo: Ediciones de la Universidad de Oviedo, 2015: 355-364.

Saneleuterio, Elia. Plagiarism in university academic papers. En INTED 2012 Proceedings. Valencia: IATED, 2012: 428-434.

Saneleuterio, Elia. Práctica de iniciación en búsquedas bibliográficas. En \#02 Jornadas de Innovación Docente en la Educación Superior. Catarroja: Florida Universitària, 2014: 327-334.

Servicio de Biblioteca Universidad de la Laguna. Estilos de cita. Cómo buscar información (2015). Recuperado de http://www.ull.es/view/institucional/bbtk/Estilos_de_cita/es

Sutherland-Smith, W. Plagiarism, the internet and Student Learning: Improving Academic Integrity. Abingdon: Taylor and Francis, 2008.

Zerdán, C. P. Metadiscurso y producción escrita. Educación, Lenguaje y Sociedad, 1.1 (2003): 221-236. 